\title{
Uncertain LTI-models for Linear Control Design of Non-Linear Systems
}

Torkel Glad, Anders Helmersson, Lennart Ljung

Division of Automatic Control

E-mail: torkel@isy.liu.se, andersh@isy.liu.se, ljung@isy.liu.se

29th June 2007

Report no.: LiTH-ISY-R-2808

Accepted for publication in Proc. Conference on Decision and Control, Orlando, FL 2001

Address:

Department of Electrical Engineering

Linköpings universitet

SE-581 83 Linköping, Sweden

WWW: http://wWw.control.isy.liu.se

AUTOMATIC CONTROL

REGLERTEKNIK

LINKÖPINGS UNIVERSITET

Technical reports from the Automatic Control group in Linköping are available from http://www. control.isy.liu.se/publications. 


\begin{abstract}
Much attention in robust identification and control has been focused on linear low order models approximating high order linear systems. We consider the more realistic situation with a linear model approximating a non-linear system. We describe how a linear time invariant (LTI) model with unstructured uncertainty, i.e. a "band" of Nyquist curves can be developed using a non-linear model error model. Applying standard linear robust control design to this uncertain LTI model will lead to a (non-linear) closes loop system with performance robustness guarantees (in terms of gain from disturbances to output) well in line with the objectives of the linear design. Clearly the design can be successful only if the linear model is a reasonable good approximation of the system. A particular aspect of the design process is to define a workable definition of "practical stability" for robust control design, with possibly non-linear model errors. We use affine power norms for that purpose.
\end{abstract}

Keywords: robustness, nonlinear system, model approximation 


\title{
Uncertain LTI-models for Linear Control Design of Non-Linear Systems
}

\author{
S. T. Glad, A. Helmersson and L. Ljung \\ Department of Electrical Engineering \\ Linköping University \\ S-581 83 Linköping, Sweden
}

\begin{abstract}
Much attention in robust identification and control has been focused on linear low order models approximating high order linear systems. We consider the more realistic situation with a linear model approximating a non-linear system. We describe how a linear time invariant (LTI) model with unstructured uncertainty, i.e. a "band" of Nyquist curves, can be developed using a non-linear model error model. Applying standard linear robust control design to this uncertain LTI model will lead to a (non-linear) closed loop system with performance robustness guarantees (in terms of gain from disturbance to output) well in line with the objectives of the linear design. Clearly the design can be successful only if the linear model is a reasonably good approximation of the system. A particular aspect of the design process is to define a workable definition of "practical stability" for robust control design, with possibly non-linear model errors. We use affine power norms for that purpose.
\end{abstract}

\section{Introduction}

In this paper we consider the estimation of linear models, of the type

$$
y(t)=G(q, \theta) u(t)+H(q, \theta) e(t)
$$

while keeping in mind that the true system will probably lie outside the model class, for instance because it contains a nonlinearity. The standard LTI identification machinery, e.g., [6], gives a transfer function estimate

$$
\hat{G}_{N}(q)=G\left(q, \hat{\theta}_{N}\right)
$$

along with an uncertainty region $\mathcal{G}$ defined by a transfer function $W(q)$ :

$$
\mathcal{G}=\left\{G \| G\left(e^{i \omega}\right)-\hat{G}_{N}\left(e^{i \omega}\right) \mid \leq W\left(e^{i \omega}\right), \forall \omega\right\}
$$

This can be used to design a regulator

$$
u(t)=F(q)(r(t)-y(t))
$$

so that the closed loop system

$$
y(t)=\frac{F(q) \hat{G}_{N}(g)}{1+F(q) \hat{G}_{N}(q)}
$$

behaves well. The design has to take the uncertainty region $\mathcal{G}$ into account. The extensive literature on robust control design, e.g., [15], [12] deals with the question to ensure good behavior of the closed loop system, despite inaccuracies in the model $\hat{G}_{N}$.

An important question is how to find the uncertainty region. The standard LTI-machinery delivers bounds that are based on estimates of the statistical uncertainty, i.e. the variance of the estimated parameters. This assumes that the model class indeed contains a "true" description of the system.

The interplay between the estimation and the design processes has received substantial attention over the past years, e.g., [4], [11], [3], [5], [13], [10].

Part of this problem is to estimate, in reliable way, the model error or model uncertainty, not assuming that the true system can be described within the chosen structure (1). See, e.g., [9], [8]. Then it is mostly assumed that the true system is linear, but of higher order than the model. In this contribution we shall consider the more general situation of possibly non-linear model errors, and discuss the roles that an explicit model error model will play in such a set-up, to define an equivalent uncertain LTI model (3). For simplicity we confine ourselves to single-input-single-output systems. See also [7]

\section{Model Error Models and Their Gains}

A model error model is a description of how the input $u$ affects the output error of the nominal model $\varepsilon$. We will typically not be interested in a detailed such description - that should rather be done as a more detailed nominal model. Primarily we focus on bounds on the gain of such a model. To go well together with linear control design, we shall work with combinations of frequency weightings and unstructured, unknown models with bounded (estimated) gain. That means that the general structure of the model from $u$ to $\varepsilon$ can be depicted as in Figure 1. Formally we have 


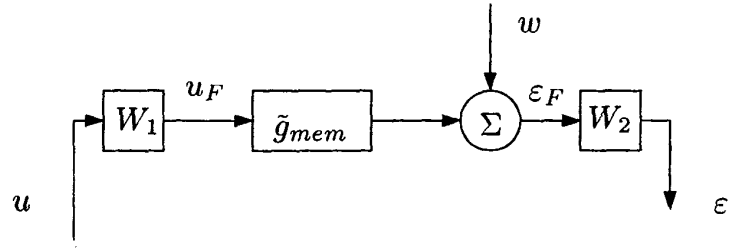

Figure 1: The model error model

$$
\begin{aligned}
& \varepsilon_{F}(t)=W_{2}^{-1}(q) \varepsilon(t), \quad u_{F}(t)=W_{1}(q) u(t) \\
& \varepsilon_{F}(t)=\tilde{g}_{m e m}\left(u_{F}^{t-1}\right)+w(t)
\end{aligned}
$$

Here, $W_{1}$ and $W_{2}$ are given linear filters, superscript $t-1$ denotes all the signal values up to time $t-1$, and $\tilde{g}_{m e m}$ is a general dynamic model. The bottom line is that, with some amount of confidence, based on the measured data, and possibly on prior information, we should be able to estimate its gain.

\section{The nonlinear gain definition}

An important aspect of nonlinear model error models is that the standard definition of gain for a nonlinear system with input $u$ and output $y$ :

$$
\sup _{u \neq 0} \frac{\|y\|}{\|u\|}
$$

is sometimes misleading. Consider for instance a static nonlinearity with a small relay-type nonlinearity at the origin. The gain becomes $+\infty$ no matter how small $\epsilon$ is. This type of nonlinearity is quite common in actuators and power amplifiers where positive and negative signals are handled by different physical components, that are not perfectly matched.

We will instead use an affine version of the gain definition. This has been used in the nonlinear control literature, see e.g. Vidyasagar [14]. We use the power gain definition of $[2,1]$ and say that the nonlinear system has gain $\beta$ with offset $\alpha$ if there are positive constants $\alpha$ and $\beta$ such that

$$
\int_{0}^{\tau} y^{2}(t) d t \leq \alpha \tau+\beta^{2} \int_{0}^{\tau} u^{2}(t) d t
$$

for all input-output pairs $u$ and $y$ and all positive $\tau$.

A discussion of the feasibility to estimate $\alpha$ and $\beta$ in (9) is given in [7].

\section{Control design}

Consider the system of figure 2 . It consists of a nominal linear model $G_{\text {nom }}$ together with the nonlinear system $y_{m e m}$ allu une intear welgins (transier lunciuns) $v v_{1}$ and $W_{2}$. It is controlled using linear feedback via the transfer function $F$. We assume that there is a known

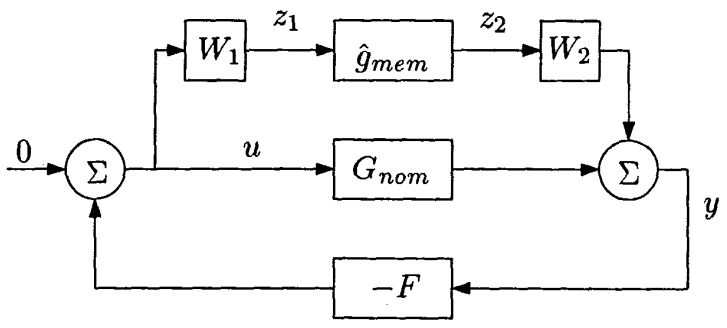

Figure 2: Block diagram of feedback loop with model error

affine gain limit on $\hat{g}_{m e m}$ so that

$$
\int_{0}^{\tau} z_{2}^{2}(t) d t \leq \alpha \tau+\beta^{2} \int_{0}^{\tau} z_{1}^{2}(t) d t
$$

For this linear nominal system with its (possibly) nonlinear model error model we assume that the uncertain LTI model (3) is delivered with $\hat{G}_{N}=G_{n o m}$ and $W=$ $\beta W_{1} W_{2}$. We should also think of $W_{2}$ as a shaping filter for the external disturbances at the output.

Applying standard robust linear control design will lead to a regulator $F$ with sensitivity $(S)$ and complementary sensitivity $(T)$ functions of the closed loop system (without the error model):

$$
S=\frac{1}{1+G_{n o m} F}, \quad T=\frac{G_{n o m} F}{1+G_{n o m} F}
$$

such that $\left\|S W_{2}\right\|_{\infty}$ is small as such that the ratio between the $T$ and the "normalized relative model error"

$$
G_{w}=T \frac{W_{1} W_{2}}{G_{n o m}}
$$

has a gain less than $1 / \beta$. We now have the following property of the closed loop system.

Proposition 1 Let the system be described by Figure 2. Assume that $\beta\left\|G_{w}\right\|<1$. Then the output $y$ is bounded by

$$
\int_{0}^{\tau} y^{2}(t) d t \leq\left\|S W_{2}\right\|^{2} \frac{\alpha \tau}{1-\beta^{2}\left\|G_{w}\right\|^{2}}
$$

Proof: Following the signals around the block diagram of Figure 2 gives the relations

$$
\begin{aligned}
z_{1} & =-G_{w} z_{2} \\
y & =S W_{2} z_{2}
\end{aligned}
$$

The relation (10) together with (11) then gives

$$
\int_{0}^{\tau} z_{2}^{2} d t \leq \frac{\alpha \tau}{1-\beta^{2}\left\|G_{w}\right\|^{2}}
$$


Froin (1८) we gel

$$
\int_{0}^{\tau} y^{2} d t \leq\left\|S W_{2}\right\|^{2} \frac{\alpha \tau}{1-\beta^{2}\left\|G_{w}\right\|^{2}}
$$

Consequently, the robust linear control objectives to shape $S$ for noise reduction at the same time as $T$ is shaped for robustness will also guarantee a good disturbance rejection in the true, nonlinear closed loop system.

\section{Discussion}

Of course it is impossible to give absolute robustness guarantees for a design based only on identification data (and hence on a finite number of limited precision measurements) and with no a priori knowledge of the physical system. Proposition 1 shows that a precise robustness result can be proven if the parameters $\alpha$ and $\beta$ of the affine gain of the model error model are known. This shows that one aim of the identification procedure could be to give as reliable estimates as possible for those parameters. Note that the result of the proposition does not give asymptotic stability in the case of $\alpha>0$. Instead an upper bound on $\|y\|$ is given.

The standard LTI paradigm of estimating linear models always gives an uncertainty set around a linear system (the second order equivalent). The size of this set tends to zero as more an more measurements become available, [7]. This is an unrealistic aspect of LTI-modeling: the system will not be exactly known even with very many observations. The way to define an LTI uncertainty set $\mathcal{G}$ as in (3) with $W=\beta W_{1} W_{2}$ from Figure 2 and (10) will allow some remaining uncertainty even when the number of data tends to infinity. If this remianing uncertainty is too large to handle with linear robust control theory, we have an indication that a non-linear regulator is required.

On the other hand, if the uncertainty region is small enough to allow for robust linear control, we have seen that applying standard, linear loop shaping of $S$ and $T$ from $\hat{G}_{N}, \beta W$ and $W_{2}$ will lead to a design that also handles the nonlinearities in a nice fashion.

An uncertain LTI model, constructed as described here, can thus very well be used for linear design, even if the true system is nonlinear. This is of course also evident from all real applications done with robust control theory, since no real life system is exactly LTI.

A key problem is to estimate the gains in (10). Some preliminary tests were made in [7], but this is a topic that needs further research.

\section{neierences}

[1] Peter M. Dower. Comments on $l_{2}$-gain analysis of systems with persistent outputs. In Proceedings of the 39th CDC, pages 263-268, 2000.

[2] Peter M. Dower and Matthew R. James. Stability of nonlinear systems with worst case power gain disturbances. In Proceedings of the 37th $C D C$, pages 4075-4080, 1998.

[3] Michel Gevers. Towards a joint design of identification and control? In $\mathrm{H} \mathrm{L}$ Trentelman and $\mathrm{J} \mathrm{C}$ Willems, editors, Essays on control: Perspectives in the theory and its applications, ECC '93 Groningen, 1993.

[4] R. Kosut, M. K. Lau, and S. P. Boyd. Setmembership identification of systems with parametric and nonparametric uncertainty. IEEE Trans. Automatic Control, AC-37:929-941, 1992.

[5] R.L. Kosut, G. C. Goodwin, and M. P. Polis (Eds). Special Issue on System Identification for Robust Control Design, IEEE Trans. Automatic Control, Vol 37. 1992.

[6] L. Ljung. System Identification - Theory for the User. Prentice-Hall, Upper Saddle River, N.J., 2nd edition, 1999.

[7] L. Ljung. Estimating linear time invariant models of non-linear time-varying systems. European Journal of Control, ((To appear)), Sept 2001. Semi-plenary presentation at the European Control Conference, Sept 2001.

[8] Lennart Ljung. Model validation and model error modeling. In B. Wittenmark and A. Rantzer, editors, The Åström Symposiium on Control, pages 15 -42, Lund, Sweden, Aug 1999. Studentlitteratur.

[9] B. Ninness and G. C. Goodwin. Estimation of model quality. Automatica, 31(12):1771-1797, 1995.

[10] K. Poolla, P. P. Khargonekar, A. Tikku, J. Krause, and K.Nagpal. A time-domain approach to model validation. IEEE Trans. on Automatic Control, AC-39:951-059, 1994.

[11] S. Rangan and K. Poolla. Time-domain validation for sample-data uncertainty models. IEEE Trans. Automatic Control, AC-41:980-991, 1996.

[12] S. Skogestad and I. Postlethwaite. Multivariable Feedback Control. John Wiley, New York, 1996.

[13] R. Smith and G.E. Dullerud. Continuous-time control model validation using finite experimental data. IEEE Trans. Automatic Control, AC-41:1094-1105, 1996.

[14] M. Vidyasagar. Nonlinear Systems Analysis. Prentice Hall, 1993.

[15] K. Zhou, J. C. Doyle, and K. Glover. Robust and Optimal Control. Prentice-Hall, Englewood Cliffs, NJ, 1996. 


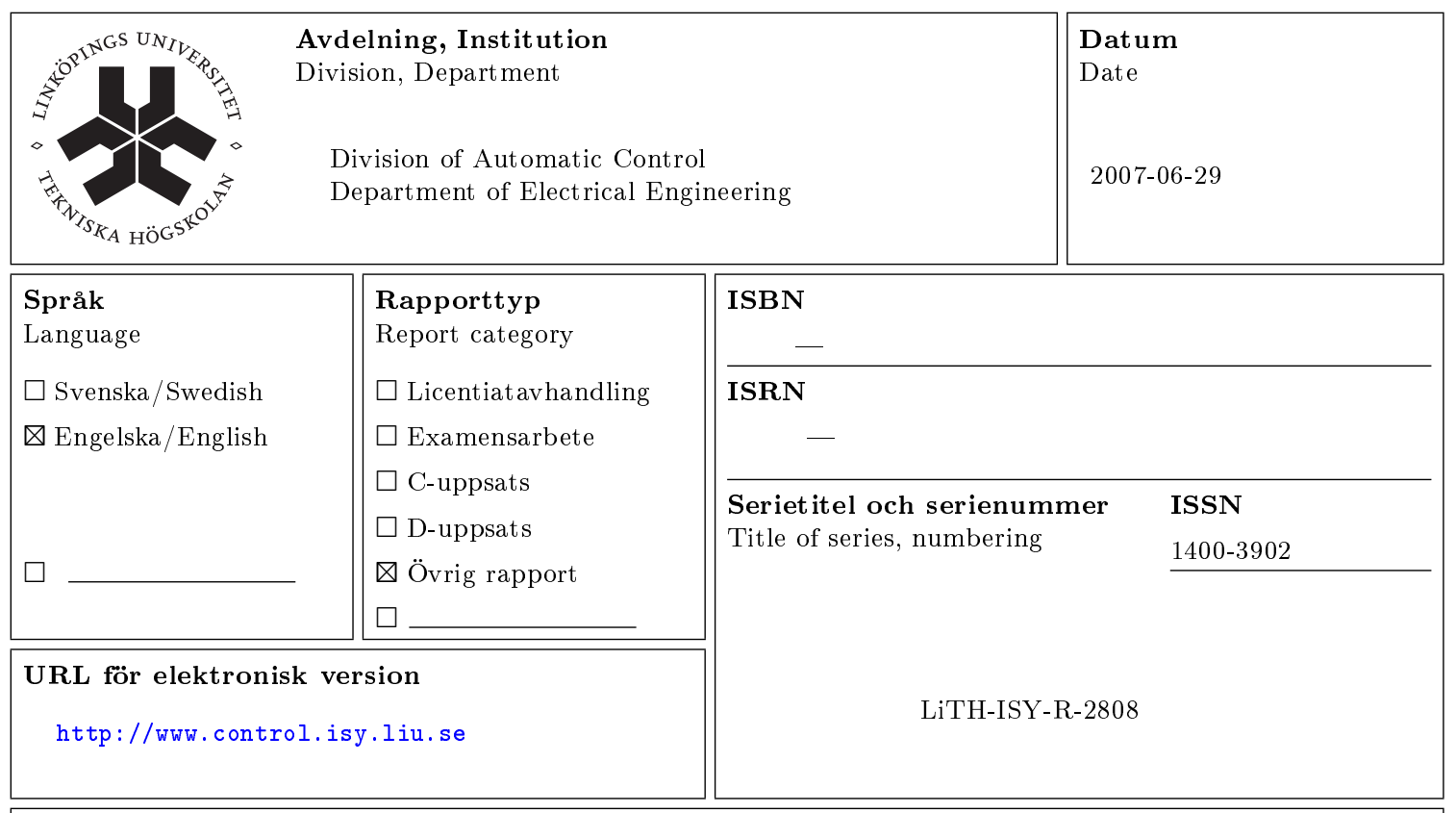

Titel Uncertain LTI-models for Linear Control Design of Non-Linear Systems

Title

Författare Torkel Glad, Anders Helmersson, Lennart Ljung

Author

\section{Sammanfattning}

Abstract

Much attention in robust identification and control has been focused on linear low order models approximating high order linear systems. We consider the more realistic situation with a linear model approximating a non-linear system. We describe how a linear time invariant (LTI) model with unstructured uncertainty, i.e. a "band" of Nyquist curves can be developed using a non-linear model error model. Applying standard linear robust control design to this uncertain LTI model will lead to a (non-linear) closes loop system with performance robustness guarantees (in terms of gain from disturbances to output) well in line with the objectives of the linear design. Clearly the design can be successful only if the linear model is a reasonable good approximation of the system. A particular aspect of the design process is to define a workable definition of "practical stability" for robust control design, with possibly non-linear model errors. We use affine power norms for that purpose. 